\title{
Anti-GAD Cerebellar Ataxia Associated with Neuroendocrine Pancreatic Tumor, Diagnostic and Therapeutic Approach
}

\author{
Anfal Al Raisi ${ }^{1}$, Sultan Al Mazroui ${ }^{1}$, Ahmed Sameer Nadeem ${ }^{2 *}$ and Abdullah AL Salti ${ }^{2}$ \\ ${ }^{1}$ Oman Medical specialty board, GFP program, Oman \\ ${ }^{2}$ Department of Neurology, Khoula Hospital-MOH, Oman
}

Submission: October 23, 2019; Published: November 12, 2019

*Corresponding author: Ahmed Sameer Nadeem, Department of Neurology, Khoula Hospital-MOH, Oman

\begin{abstract}
Anti- Glutamic Acid Decarboxylase antibodies (anti-GAD Abs) associated Cerebellar Ataxia (CA) is one of the rare neurologic conditions that affect the central nervous system and has been linked to autoimmune endocrine disorders or associated with paraneoplastic tumors [13]. Here we report a case of a 37 years old Omani woman, presented with symptoms of subacute cerebellar ataxia, with high serum levels of anti-Glutamic acid decarboxylase antibodies which suggests the diagnosis of Anti- Glutamic acid decarboxylase antibodies cerebellar ataxia. Since there are no approved international guidelines for the treatment of immune-mediated anti-GAD cerebellar ataxia and the management is case dependent [4], we used a trial of combination of maintenance therapies, after induction with a 5 days course of mehylpredinisolon and tapering, including monthly IV Immunoglobulin, followed by adding a course of Rituximab and daily Azathioprine for 12 months, which lead to symptomatic improvement. One year follow up with PAN CT scan (chest, abdomen and pelvice) showed a neuroendocrine panceatice tumor with no metastasis, which has been totally resected. The patient dramatically improved, with no further medications, despite the persistence high anti GAD antibodies titer. We concluded that the triple maintenance therapy used is highly effective and led to significant clinical improvement; in addition, there is no correlation between the clinical, as well as the symptomatic improvements and the anti-GAD Antibodies titers. Moreover, we highly recommend repeating tumor workup, serology and imaging, at least after one year of presentation, if malignancy is highly suspicious.
\end{abstract}

Keywords: Anti- Glutamic acid decarboxylase antibodies (Anti-GAD Abs); Cerebellar ataxia; Pancreatic tumor; Paraneoplastic syndrome

Abbreviations: CFS: Cerebral-Spinal Fluid; SPS: Stiff Person Syndrome; GAD: Glutamic Acid Decarboxylase; CA: Cerebellar Ataxia; IVIG: Intravenous Immunoglobulin; GABA: Gamma-Aminobutyric Acid

\section{Introduction}

Glutamic Acid Decarboxylase (GAD) is a $\gamma$-Amino Butyric Acid (GABA) synthesizing enzyme found in the nervous system and the pancreas. Lacking this enzyme can lead to epilepsy, Stiff Person Syndrome (SPS) [1], limbic encephalitis [5] and Type 1 DM [6]. The association between GAD-Abs and SPS is well established and there is arising evident of the association between GAD-antibodies and CA [7,8]. High titers of antiGAD Abs can present with various neurological syndromes. Therefore, it has been challenging to diagnose isolated Anti- GAD $\mathrm{CA}$, as there are no standardized clear international guidelines in managing such cases [4]. In this paper we are interested in sharing our experience in the diagnosis and treatment approach, with a triple maintenance therapy, for managing a case of anti-GAD Abs cerebellar ataxia, which led to significant improvement of symptoms. Reported cases of autoimmune cerebellar ataxia show significant neurological improvement using; Corticosteroids [9], Intravenous Immunoglobulin (IVIG) [10], Immunosuppressants and Plasma exchange [11,12]. With paraneoplastic tumors, tumor resection usually plays a major role in improving the symptoms.

\section{Case Report}

A 37-years-old Omani woman, not known to have any past medical history developed gradual sub-acute onset of dizziness associated with unsteadiness and blurry vision that caused recurrent falls. Subsequently, she had intermittent nausea, vomiting and oscillopsia. Symptoms exacerbated with movement, progressing over time and led her to use walking aid. The symptoms significantly affected her daily activities. The patient reported $10 \mathrm{~kg}$ weight loss in 6 months, with no history of changes in bowel habits. Furthermore, there was neither 


\section{Open Access Journal of Neurology \& Neurosurgery}

history of any impairment in cognitive functions, psychiatric symptoms, seizures, ocular-bulbar symptoms, abnormal body movements, muscular weakness nor any sensory abnormalities. Her systemic review is unremarkable, no family history of autoimmune diseases and there is no history of recent vaccination or travel.

Neurological examination disclosed; normal higher mental function, with bilateral horizontal nystagmus, bilateral Dysmetria, dysdiadochokinesia more on the right side. Moreover, the patient had intention tremor, positive pendular knee reflex, positive heel-knee shin test, wide-based ataxic gait, and severely impaired tandem gait. However, there was a normal tone, power, negative Babinski's sign and all Sensory modalities' examination tests were bilaterally intact. Our case presented an isolated sub-acute cerebellar ataxia which was investigated for any acquired (acute and sub-acute) conditions. Basic routine blood work-up was normal. Urgent contrast MRI/MRA/ MRV brain followed by spine MRI to rule out space occupying lesion (such as malignancy), infarction, demyelinating diseases, vasculitis or any inflammatory and infectious causes. All radiological investigations showed normal brain and spine images with no evidence of cerebellar atrophy. Cerebral-Spinal Fluid (CSF) cell count, protein \& glucose content were normal. PCR analysis of the CSF was done for herpes simplex, varicella zoster, mumps and enterovirus and none was detected. Laboratory test was done to rule out acute celebrealities (post viral, Lyme, HIV, TB, .etc), and came with unremarkable results. Nerve conduction study and EEG testing results were also normal.

At this time, she was treated empirically with Methylprednisolone 1gm, once daily for 5 days and symptomatic treatment for vomiting. Serological studies for auto antibodies; Anti-Tissue Transglutaminase antibodies (TTG-IgA) and esophageal-Gastro-Duodenoscopy (OGD) with duodenal biopsy were unremarkable; therefore, celiac disease was ruled out. Antithyroid antibodies, thyroglobulin antibodies, thyroid peroxidase antibodies, anti-islet cells antibodies, rheumatoid factor, were done and all results were negative. Subsequently, Hashimoto's thyroiditis, Graves' disease, and rheumatoid arthritis were excluded.

Sent as a part of auto-immune work up, serum Anti-GAD antibodies titers result was highly positive, > $2000 \mathrm{U} / \mathrm{mL}$ (our laboratory normal range: $<10 \mathrm{U} / \mathrm{mL}$ ). Therefore, extended laboratory workup was done to exclude paraneoplastic syndromes, including tumor markers; Cancer antigen 19.9, CA 125, Alpha-feto protein, as well as Carcino Embryogenic Antigen; all results were normal. As part of tumor workup, PAN CT \& PET scans imaging were done and reported as normal without detecting any mass or tumor growth. Therefore, the patient was diagnosed with autoimmune Anti-GAD Abs cerebellar ataxia.

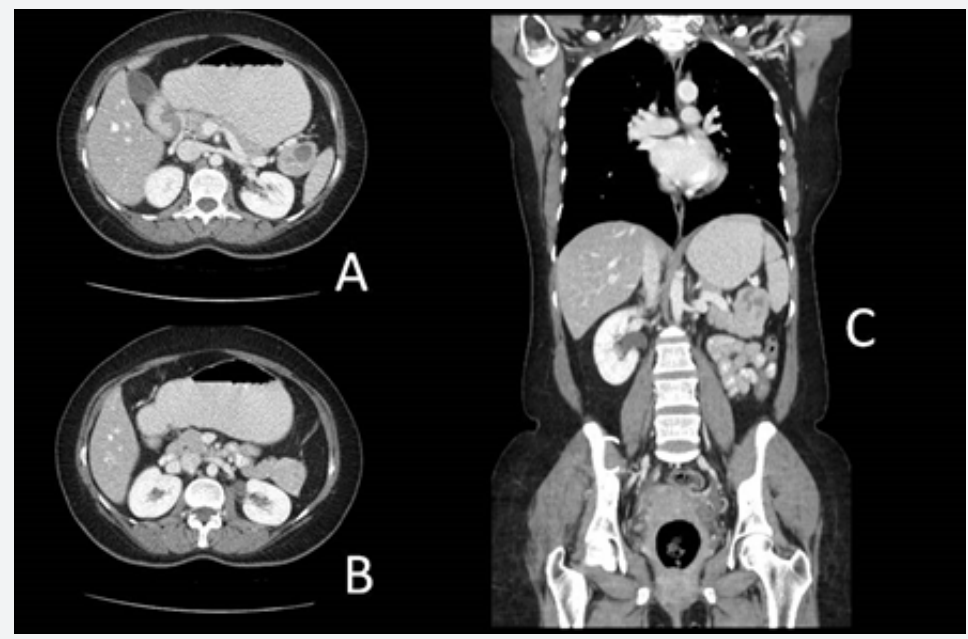

Figure 1: Axial Contrast-Enhanced CT Scan Examination (Image A, and Image B) With Coronal Reformatted Image,(Image C), In the Early Portovenous Phase Demonstrates A Mass With Cystic Components in the Tail of the Pancreas, which is Isodense Compared to the Pancreatic Parenchyma, Denoting its Relative Hypervascularity, and no Associated Pancreatic Duct Dilatation Seen.

Since the management of anti-GAD cerebellar ataxia is case based and there are no clear treatment guidelines [4], we started a trail of triple maintenance therapy, after tapering the initial steroid therapy, which resulted in a significant clinical response. A course of monthly 25 grams IV immunoglobulin and $1000 \mathrm{mg}$ Rituximab given at day 1 and day 15 with a daily dose of $50 \mathrm{mg}$ Azathioprine, for 2 weeks, then increased to $100 \mathrm{mg}$ for another 2 weeks, followed by a fixed daily dose of $150 \mathrm{mg}$. After 6 months of initiating the treatment, the patient showed significant improvement in symptoms. She recovered from vertigo, nausea and vomiting, had considerable improvement in gait, as she could walk independently using walking aid occasionally. However, mild blurred vision and mild bilateral nystagmus persisted. The patient continued treatment with this clinically significant moderate improvement. However, a follow up of her anti-GAD Abs titer after 1 year of treatment was still persistently high, > $2000 \mathrm{U} / \mathrm{mL}$, regardless of the remarkable clinical improvements, therefore, a second CT abdomen was done and showed $4.5 \mathrm{~cm}$ 


\section{Open Access Journal of Neurology \& Neurosurgery}

enhancing lobulated mass with internal cystic component at the pancreatic tail with no lymphadenopathy and no evidence of metastatic lesions (Figure 1). Thus, the patient underwent open distal pancreatectomy and splenectomy. Histopathological report showed neuroendocrine tumor grade II (Figure 2). The patient was discontinued from medical treatment and she recovered well from the surgery. There was dramatic added symptomatic and clinical improvement to her residual cerebellar symptoms.

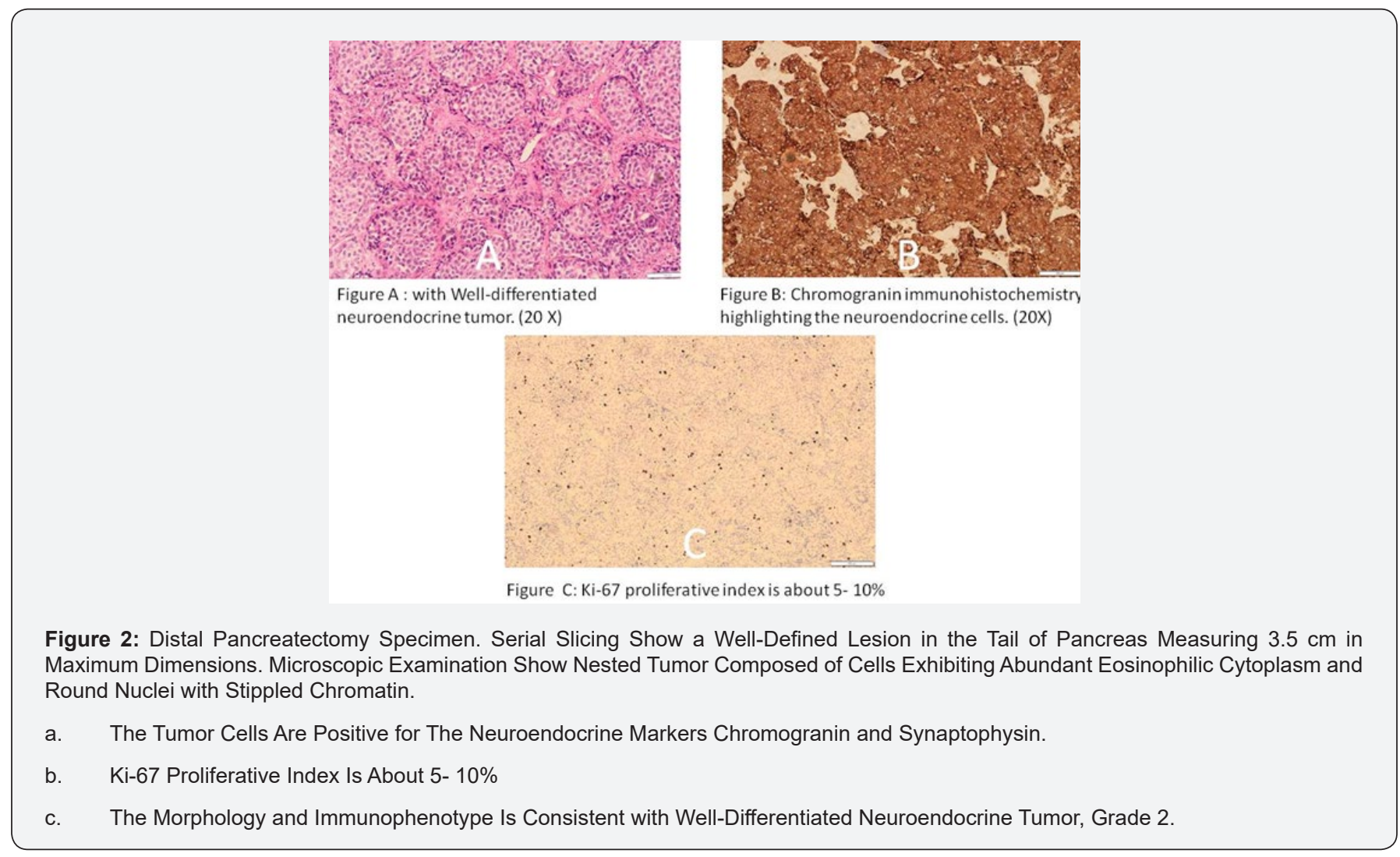

The patient was assessed after 8 months of the surgical resection of the tumor, and she showed significant improvement of her symptoms, even though she was not on any medical treatment. She is thriving well, gained the weight that she had lost but only left with occasional oscillopsia and unsteadiness while walking for long distance. Clinically, she is now able to walk unassisted with wide based gait, has high amplitude slow gaze vertical nystagmus and her dysmetria is limited to the right side, no dysdiadochokinesis, other neurological examination is unremarkable. The patient's anti-GAD Abs titer remained high, but her Chromogranin-A serum level decreased from $108 \mathrm{U} / \mathrm{ml}$ to normal levels after surgical intervention, and the octreotide scintigram result was negative pre and post-surgical resection.

\section{Discussion}

Anti-GAD Abs cerebellar ataxia is a rare form of cerebellar ataxia which commonly affects woman whom are diagnosed with other autoimmune conditions [13]. The Diagnosis of AntiGAD Abs cerebellar ataxia is made based on obtaining high levels of serum anti-GAD antibodies [1] which is often either associated with autoimmune or paraneoplastic syndromes; such as neuroendocrine tumor; lungs squamous cell carcinoma, thymomas, breast, hematological,..etc. [14,15]. Glutamic Acid Decarboxylase (GAD) is a neuronal enzyme involved in the synthesis of the neurotransmitter Gamma-Aminobutyric Acid (GABA). It is expressed in the CNS neuronal cells as an inhibitory neurotransmitter which reduces the neuronal excitability. Thus, reduction in GABA levels in the brain can lead to increase firing of nerve cells leading to conditions like anxiety, CA, and seizure disorders [5]. Although the exact pathogenic effect of Anti-GAD Abs is still unclear and controversial, their effect on Purkinje cells have been demonstrated experimentally causing direct excitotoxicity $[16,17]$.

Anti-Glutamic acid decarboxylase antibodies appear in patients with Stiff Person Syndrome (SPS) [18,19], Cerebellar ataxia and other neurological disorders, including progressive encephalomyelitis with rigidity and myoclonus, epilepsy, limbic encephalitis, and paraneoplastic syndromes [1,18-20]. These antibodies are also associated with the risk of other autoimmune disorders such as Thyroid disease, Celiac disease [4] and lateonset type 1 Diabetes Mellitus [6]. Therefore, most of the reports of autoimmune CA were in patients who had other associated autoimmune conditions, such as late onset diabetes militias, Sjogren syndrome, Graves' disease, and Hashimoto's thyroiditis, and it's very rare to find an isolated cerebellar ataxia. SPS is the highest associated neurological disorder with positive GAD-Abs, followed by CA $[5,12]$. 
There are only two reported cases of paraneoplastic pancreatic tumor, the first case is a 67 year old male presented with encephalomyelitis [21], the second case is a 61 years old male patient with DM type 2, presented with symptoms of acute cerebellar degeneration, with normal initial lab tests and radiological investigation. A follow up of abdominal CT scan showed a large pancreatic mass with multiple liver metastases and the histopathology report showed a well-differentiated neuroendocrine tumor. The patient was started on monthly intramuscular corticosteroid with daily oral prednisone. The patient initially showed obvious neurological improvement in the first three months, but later on he started deteriorating requiring hospital admission, and he died due to neurological impairment after seven months of the onset of the symptoms [3].

Cerebellar dysfunction with GAD-Abs is recognized as an autoimmune disorder that can be treated [8]. However, there is no clear treatment guideline for anti-GAD Abs cerebellar ataxia as and the management is case based dependent. Lowering the autoimmune activity is the aim of the treatment. The treatment composes of surgical resection of the tumor [22], if it was associated with paraneoplastic tumor, and medical management [4]. The medical treatment composes of Intravenous immunoglobulin (IVIG), Steroids, Rituximab and oral immunosuppressive drugs $[4,17]$. In our case, as a first line treatment, a combination of IVIG and Rituximab was initiated, associated with a maintenance therapy with Azathioprine 150 $\mathrm{mg} /$ day which led to some clinical improvement. Sub-acute CA with positive anti-GAD Abs has good prognosis if immediate immune therapy has been started [8]. During the patient's follow up, a pancreatic tumor was discovered which was totally removed surgically with no complications. After the surgical intervention, further and dramatic symptomatic improvement was seen. In spite of tumor resection and continuation of immunotherapy, her anti GAD Abs titer is still high. However, she has shown impressive symptomatic and clinical improvement which may be explained by an intra thecal antibody production [22]. Avoiding the delay to initiate the treatment with immunotherapy in cases of sub-acute cerebellar ataxia has certainly good prognosis, especially if there is no evidence of cerebellar atrophy [17]. In this case report, we are sharing the effectiveness of triple maintenance therapy in addition to tumor resection. The medical management includes; Rituximab, monthly IVIG and daily Azathioprine. The patient has remarkable clinical improvement; she is back to her work with minimal gait ataxia regardless of the persistent high antibodies titer.

\section{Conclusion}

The main treatment of paraneoplastic syndrome is tumor resection followed by immunosuppressant. However, there are no clear management guidelines of autoimmune cerebellar ataxia, as there are various subtypes of immune mediated cerebellar ataxia. We have found that the triple maintenance therapy used is highly effective and led to significant clinical improvement to our patient. In addition, we concluded that there is no correlation between the clinical, as well as the symptomatic improvements and the anti-GAD Abs titers. In addition, we highly recommend repeating tumor workup; serology and imaging, at least after one year of presentation, if malignancy is highly suspicious.

\section{Acknowledgment}

We would like to express our sincere gratitude and appreciation to Mr. Mohammed Al Mammari at the National University Sohar-Oman, Dr. Atheel Ahmed-Radiology Department and to Dr. Mohammed Almasqari-Histopathology Department at the Royal hospital-Oman for their valuable contribution and guidance that greatly assisted the completion of this case report.

\section{Consent}

Informed consent for the publication of the clinical details, tests' results and the radiological images was obtained from the patient and documented in the patient's electronic file.

\section{Competing Interest}

The authors declare no financial competing interest. None of the authors received reimbursements, fees, funding, or salary from an organization that may in any way gain or lose financially from the publication of this manuscript, either now or in the future. The authors declare no non-financial competing interests (personal, ideological, academic, intellectual, commercial or any other) in relation.

\section{References}

1. Saiz A, Blanco Y, Sabater L, González F, Bataller L, et al. (2008) Spectrum of neurological syndromes associated with glutamic acid decarboxylase antibodies: diagnostic clues for this association. Brain 131(10): 2553-2563.

2. Graus F, Saiz A, Dalmau J (2010) Antibodies and neuronal autoimmune disorders of the CNS. J Neurol 257: 509-517.

3. Brizzi MP, Sonetto C, Tampellini M, Di Maio M, Volante M, et al. (2015) Unusual paraneoplastic neurological syndrome secondary to a well differentiated pancreatic neuroendocrine tumor: a case report and review of the literature. BMC cancer 15: 914.

4. Mitoma H, Hadjivassiliou M, Honnorat J (2015) Guidelines for treatment of immune-mediated cerebellar ataxias. Cerebellum \& ataxias 2: 14.

5. Tohid H (2016) Anti-glutamic acid decarboxylase antibody positive neurological syndromes. Neurosciences 21(3): 215-222.

6. Tuomi T, Groop L, Zimmet P, Rowley M, Knowles W, et al. (1993) Antibodies to glutamic acid decarboxylase reveal latent autoimmune diabetes mellitus in adults with a non-insulin-dependent onset of disease. Diabetes 42(2): 359-362.

7. Raju R, Foote J, Banga J, Hall T, Padoa C, et al. (2005) Analysis of GAD65 Autoantibodies in Stiff-Person Syndrome Patients. The Journal of Immunology 175(11): 7755-7762.

8. Ariño H, Gresa-Arribas N, Blanco Y, Martínez-Hernández E, Sabater L, et al. (2014) Cerebellar Ataxia and Glutamic Acid Decarboxylase Antibodies: immunologic profile and long-term effect of immunotherapy. JAMA Neurology 71(8): 1009.

9. Lauria G, Pareyson D, Giuseppina pitzolu M, Bazzigaluppi E (2003) Excellent response to steroid treatment in anti-gad cerebellar ataxia: Lancet neurol 2(10): 634-635. 
10. Abele M, Weller M, Mescheriakov S, Bürk K, Dichgans J, et al. (1999) Cerebellar ataxia with glutamic acid decarboxylase autoantibodies: Neurology 52(4): 857-859.

11. Vulliemoz S, Vanini G, Truffert A, Chizzolini C, Seeck M, et al, Epilepsy and cerebellar ataxia associated with anti-glutamic acid decarboxylase antibodies. J Neurol Neurosurg Psychiatry 78(2): 187-189.

12. Georgieva Z, Parton M (2014) Cerebellar ataxia and epilepsy with anti-GAD antibodies: treatment with IVIG and plasmapheresis. BMJ Case Rep: bcr2013202314

13. Ariño H, Gresa-Arribas N, Blanco Y, Martínez-Hernández E, Sabater L, et al. (2014) Cerebellar Ataxia and Glutamic Acid Decarboxylase Antibodies: Immunologic Profile and Long-term Effect of Immunotherapy. JAMA Neurol 71(8): 1009-1016.

14. Ariño H, Höftberger R, Gresa-Arribas N, Martínez-Hernández E, Armangue T, et al. (2015) Paraneoplastic Neurological Syndromes and Glutamic Acid Decarboxylase Antibodies. JAMA Neurol 72(8): 874-881.

15. Vianello M, Tavolato B, Armani M, Giometto B (2003) Cerebellar ataxia associated with anti-glutamic acid decarboxylase autoantibodies. Cerebellum 2(1): 77-79.

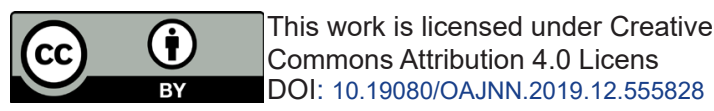

16. Baizabal-Carvallo J, Alonso-Juarez M (2017) Cerebellar disease associated with anti-glutamic acid decarboxylase antibodies: review. J Neural Transm (Vienna) 124(10): 1171-1182.

17. Dayalu P, Teener J (2013) Stiff Person syndrome and other anti-GAD-associated neurologic disorders. Semin Neurol 32(5): 544-549.

18. Leypoldt F, Wandinger KP (2014) Paraneoplastic neurological syndromes: paraneoplastic neurological syndromes. Clin Exp Immunol 175(3): 336-348.

19. Graus F, Saiz A, Dalmau J (2010) Antibodies and neuronal autoimmune disorders of the CNS. J Neurol 257(4): 509-517.

20. Hernández-Echebarría L, Saiz A, Arés A, Tejada J, García-Tuñón L, et al. (2006) Paraneoplastic encephalomyelitis associated with pancreatic tumor and anti-GAD antibodies. Neurology 66(3): 450-451.

21. Braik T, Evans AT, Telfer M, Mc Dunn S (2010) Paraneoplastic neurological syndromes: unusual presentations of cancer. A practical review. Am J Med Sci 340(4): 301-308.

22. Honnorat J, Saiz A, Giometto B, Vincent A, Brieva L, et al. (2001) Cerebellar ataxia with anti-glutamic acid decarboxylase antibodies: study of 14 patients. Arch Neurol 58: 225-230.

\section{Your next submission with Juniper Publishers will reach you the below assets}

- Quality Editorial service

- Swift Peer Review

- Reprints availability

- E-prints Service

- Manuscript Podcast for convenient understanding

- Global attainment for your research

- Manuscript accessibility in different formats

(Pdf, E-pub, Full Text, Audio)

- Unceasing customer service

Track the below URL for one-step submission

https://juniperpublishers.com/online-submission.php 